Georgia State University

ScholarWorks @ Georgia State University

\title{
Associations among perceptual anomalies, social anxiety, and paranoia in a college student sample
}

\author{
Erin B. Tone \\ Georgia State University, etone@gsu.edu
}

Follow this and additional works at: https://scholarworks.gsu.edu/psych_facpub

Part of the Psychology Commons

\section{Recommended Citation}

Tone, Erin B., "Associations among perceptual anomalies, social anxiety, and paranoia in a college student sample" (2011). Psychology Faculty Publications. 113.

https://scholarworks.gsu.edu/psych_facpub/113

This Article is brought to you for free and open access by the Department of Psychology at ScholarWorks @ Georgia State University. It has been accepted for inclusion in Psychology Faculty Publications by an authorized administrator of ScholarWorks @ Georgia State University. For more information, please contact scholarworks@gsu.edu. 
RUNNING HEAD: PERCEPTUAL ANOMALIES, SOCIAL ANXIETY, AND PARANOIA

Associations among perceptual anomalies, social anxiety, and paranoia in a college student sample

Erin Brooke Tone

Georgia State University

Sandra Goulding

Emory University

Michael Compton

Emory University

Corresponding author: Erin B. Tone, Department of Psychology, Georgia State University, P.O. Box 5010, Atlanta, Georgia, 30302-5010, USA, phone: 404-413-6291; fax: 404-413-6207; email: etone@gsu.edu. 


\begin{abstract}
Recent evidence suggests that normal-range paranoid ideation may be particularly likely to arise in individuals disposed to both social anxiety and perceptual anomalies. This study was designed to test the hypothesis that among college students in an unselected sample, social anxiety and experience of perceptual anomalies would not only each independently predict the experience of self-reported paranoid ideation, but would also interact to predict paranoid patterns of thought. A diverse sample of 644 students completed a large battery of self-report measures, as well as the five-factor Paranoia/Suspiciousness Questionnaire (PSQ). We conducted hierarchical multiple regression analyses predicting scores on each PSQ factor from responses on measures of social anxiety, perceptual aberration, and the interaction between the two constructs. Current general negative affect was covaried in all analyses. We found that both social anxiety and perceptual aberrations, along with negative affect, predicted multiple dimensions of paranoia as measured by the PSQ; the two constructs did not, however, interact significantly to predict any dimensions. Our findings suggest that perceptual aberration and anxiety may contribute to normal-range paranoid ideation in an additive rather than an interactive manner.
\end{abstract}

Keywords: Paranoid ideation, perceptual aberration, social anxiety 


\section{Introduction}

Social anxiety and paranoia are psychological phenomena whose cardinal features include expectations of negative responses from others. The nature of the anticipated negative response, however, differs subtly between the two states. Whereas excessive fear that others will evaluate one critically is at the core of social anxiety (Hofmann et al., 2004), excessive and unfounded fear of harm or maltreatment from others characterizes paranoia (Freeman, 2007). Partially underlying both types of fear are proclivities to referential patterns of thinking, or irrational, potentially delusional, beliefs that one is consistently the objects of others' notice, criticism, or blame (Meyer \& Lenzenweger, 2009; Fenigstein \& Vanable, 1992), as well as a limited capacity to evaluate the accuracy of those beliefs (Langdon \& Coltheart, 2000). However, whereas socially anxious individuals might believe that their own actions or characteristics are the root causes of the negative responses that they anticipate, paranoid individuals might instead attribute expected negative responses to active malice from others. Although both social anxiety and paranoid thinking manifest as clinically significant extremes in small proportions of the population, considerable evidence suggests that milder experiences of the two phenomena are common in the broader community (Fenigstein \& Vanable, 1992; Pollard \& Henderson, 1988).

Given their similarities it is not surprising that, although social anxiety and paranoia are typically defined as distinct constructs, recent research has found evidence of overlap between them in both psychiatric samples (Fornells-Ambrojo and Garety, 2009) and the general population (Lincoln et al., 2009). Findings from one study suggest that sub-clinical paranoid ideation may be appropriately categorized as a form of anxiety in some individuals (Freeman et al., 2008a); other research similarly links paranoid thoughts more broadly with different types of 
negative affect (NA) (Combs et al., 2007). Why only a subset of individuals with social anxiety specifically, and elevated negative affect more generally, might also demonstrate paranoid patterns of thinking, however, remains unclear. This question has provoked recent research interest and merits further investigation.

$\underline{\text { Social anxiety, perceptual anomalies, and paranoia }}$

Freeman and colleagues have recently conducted several studies examining factors that might lead some individuals, but not others, to develop paranoid patterns of thinking in the context of anxiety or broader negative affective states (Freeman et al., 2005a; Freeman et al., 2005b; Freeman et al., 2008a). Their findings converge to suggest that when individuals experience perceptual anomalies, such as sensory hypersensitivity or bodily discontinuities (i.e., feeling as if body parts are disconnected or mis-shapen), in the context of preexisting negative beliefs about themselves and others, they are more likely to interpret them as signs of persecution than they would in the absence of a negative cognitive framework.

These findings map onto a well-researched cognitive model of social anxiety (Clark \& Wells, 1995). This model postulates that socially anxious people closely monitor not only interpersonal cues, but also their own internal cues (e.g., feelings of shakiness) and use their observations to fuel negative expectations about how others will view them (Clark \& Wells, 1995; Beard and Amir, 2009). For example, a socially anxious individual who feels shaky in a feared social situation may believe erroneously that the shakiness is strikingly and embarrassingly visible to others and likely to elicit negative evaluations. It is thus plausible that socially anxious individuals who also experience perceptual anomalies have an additional, and particularly disturbing or embarrassing, set of interoceptive cues that they are vulnerable to believe others will detect and judge them for. If these individuals also exhibit biased reasoning 
processes (Garety and Freeman, 1999), low or unstable self-esteem (Ellett et al., 2003; Martin and Penn, 2001; Thewissen et al., 2007), and a proclivity to evaluate others negatively (Chadwick and Trower, 1997), paranoid ideation may be particularly likely to emerge.

These findings are also consistent with theories about delusional thinking and how it emerges. Maher (1974), for example, defined delusions as "hypothes[e]s designed to explain unusual perceptual phenomena” (p. 103). The irrational negative cognitive framework associated with social anxiety may lead affected individuals who also experience perceptual anomalies to develop subclinical, quasi-delusional beliefs that serve a similar explanatory function. Further, socially anxious individuals are prone to engage in extensive post-event processing that is colored heavily by their negative self-perceptions and that omits or downplays cues that might contradict beliefs that others evaluated them critically (Clark \& Wells, 1995). This tendency is suggestive of a limited or impaired capacity among socially anxious people to evaluate their own beliefs, a characteristic that Langdon and Coltheart (2000) proposed as an additional factor underlying the development of delusional thought.

Thus, findings from several literatures converge to suggest that the negative belief framework associated with social anxiety provides a context in which perceptual anomalies are likely to be misinterpreted as visible cues that are particularly likely to elicit, or even manifest as consequences of, harsh judgment or mistreatment by others. Further, inadequate monitoring and evaluation of beliefs among the socially anxious may perpetuate such misinterpretations and associated cognitions and emotions. Social anxiety and perceptual anomalies are thus both plausible components of mechanisms underlying the development of paranoid thought in members of the general population. 
In keeping with this idea, the present study was designed to test the hypothesis that among college students in an unselected sample, social anxiety and experience of perceptual anomalies would not only each independently predict the experience of self-reported paranoid ideation, but would also interact to predict paranoid patterns of thought. Unlike prior research focused on anxiety, perceptual anomalies, and paranoia, which has typically treated paranoia as a unidimensional construct (e.g., (Freeman et al., 2005a; Freeman et al., 2005b; Freeman et al., 2008b; Martin et al., 2001), we examined associations among social anxiety, perceptual abnormalities, and Rawlings and Freeman’s (1996) five proposed factors for sub-clinical paranoia, which they based on a factor analysis of a large item set drawn from established, theoretically-driven measures of paranoia and related constructs. We elected to use Rawlings and Freeman's (1996) model for two primary reasons. First, the Paranoia and Suspiciousness Scale (PSQ) permits separate examination of the experience of suspicion or mistrust (Suspiciousness/Hostility, Mistrust/Wariness, and Perceived Hardship/Resentment factors), which we expected to be associated with social anxiety and perceptual anomalies, as well as their interaction, and of negative emotional experiences that are not specific to paranoia (Negative Mood/Withdrawal and Anger/Impulsiveness factors). We expected Negative Mood/Withdrawal to be more strongly associated with self-reported social anxiety than with perceptual anomalies; we anticipated that neither social anxiety nor perceptual anomalies would contribute significantly to the variance in Anger/Impulsiveness when general negative affect was covaried. Second, the measure was normed on an unselected college student sample $(\mathrm{n}=561)$ that resembled our sampling population in both age and gender.

To test our hypotheses, we conducted hierarchical multiple regression analyses, in which responses on measures of social anxiety and perceptual aberration, as well as their interaction, 
were entered as predictors of scores on the five factor analysis-derived subscale scores from the Paranoia/Suspiciousness Questionnaire (PSQ) (Rawlings et al., 1996). Scores on a measure of general negative affect in the preceding week (Depression Anxiety Stress Scales; DASS;

Lovibond and Lovibond, 1995) were entered at the first step of each model as a covariate to permit examination of the specificity of associations between social anxiety and PSQ subscale scores.

2. Methods

\subsection{Subjects and Procedures}

Students enrolled in introductory psychology courses at an urban public university in the southeastern United States were invited to participate in an online survey study for course research credit. After reviewing an online informed consent form, approved by the university's Institutional Review Board, 880 students completed a series of web-based questionnaires, which were administered in randomized order across participants and typically required 30 to 60 minutes to complete ( $M=51.1$ minutes, $S D=24.1$ minutes). Participants who completed the assessment in $<20$ minutes $(n=50)$ or skipped too many items to permit calculation of total or subscale scores on any one of the four study measures were excluded ( $n=186)$, yielding a sample of 644 (as shown in Table 1, the original and final samples did not differ along any demographic variables). Participants were largely female and represented diverse racial/ethnic backgrounds. Roughly half of the participants reported that they were currently dating, engaged, or married. Past mental health treatment was endorsed by roughly a quarter of participants; depression and/or anxiety were the most commonly reported reasons (72 participants declined to report why they had received treatment).

\subsection{Measures}


Perceptual Aberration Scale (PAS; (Chapman et al., 1978): This extensively researched true/false, self-report measure consists of 35 items that focus on experience of body-image distortions and perceptual anomalies. The measure has demonstrated reliability and validity in varied samples, including college undergraduates (Chapman et al., 1995; Kwapil et al., 2008; Lenzenweger, 1994). The Cronbach's internal consistency coefficient for the PAS in the present sample was $\alpha=0.91$; Kwapil and colleagues' (2008) study yielded $\alpha=0.88$ in an undergraduate sample.

Fear of Negative Evaluation Scale (FNE; (Watson and Friend, 1969): The 30-item FNE is a broadly validated and carefully-studied self-report measure designed to evaluate apprehension about being negatively evaluated by others, a core feature of social anxiety (Orsillo, 2001; Rodebaugh et al., 2004). In prior research, estimates of internal consistency reliability have been high (KR-20 $=0.83$ - .93) (Rodebaugh et al., 2004). The Cronbach’s internal consistency coefficient for the FNE total score in the present sample was $\alpha=0.92$.

The 47-item Paranoia/Suspiciousness Questionnaire (PSQ; (Rawlings et al., 1996) is self-report scale for use in non-clinical populations that combines items from a variety of wellestablished measures of paranoia and related constructs, such as hostility. The PSQ is divided into five subscales based on factor analytic findings; these subscales are labeled as Interpersonal Suspiciousness/Hostility, Negative Mood/Withdrawal, Anger/Impulsiveness, Mistrust/Wariness, and Perceived Hardship/Resentment (Rawlings et al., 1996). The PSQ subscales have demonstrated adequate internal consistency (subscale alphas range from 0.65 to 0.77 ) and evidence suggests validity in non-clinical populations (Green et al., 2008; Gudjonsson et al., 2002). Cronbach's internal consistency coefficients for the PSQ subscales in the present sample were: Interpersonal Suspiciousness/Hostility ( 12 items) $\alpha=0.82$, Negative Mood/Withdrawal ( 7 
items) $\alpha=.58$, Anger $/$ Impulsiveness ( 9 items) $\alpha=0.70$, Mistrust/Wariness ( 6 items) $\alpha=0.74$, and Perceived Hardship/Resentment (7 items) $\alpha=0.78$.

The 42-item Depression, Anxiety, and Stress Scale (Lovibond and Lovibond, 1995) elicits ratings of depressive and anxious symptoms, as well as subjective stress, experienced during the past week. Scores on this scale correlate highly with those on widely used measures of depression and anxiety, such as the Beck Depression Inventory (Beck et al., 1996) and several well-validated anxiety questionnaires (Crawford and Henry, 2003; Lovibond \& Lovibond, 1995). The three constituent subscales (depression, anxiety, and stress) each contain 14 items, and all three have been found to have high internal consistency (Niewenhujisen et al., 2003; Sukantarat et al., 2007). In the present sample, Cronbach’s alpha coefficients for the DASS anxiety, depression, and stress subscales were $\alpha=.91, \alpha=.94$, and $\alpha=.92$, respectively.

\subsection{Statistical Analysis}

We conducted all analyses using the SPSS 18.0 program. Data were first inspected for missing data and evaluated for fit between variable distributions and the assumptions of multivariate analysis. We then centered the five predictor variables and calculated the interaction term of interest (FNE x PAS) using centered values. Finally, we conducted hierarchical multiple regression analyses examining FNE, PAS, and DASS anxiety, stress, and depression subscale scores and the interaction between FNE and PAS scores as predictors of scores on the five PSQ factor subscales. For each analysis, centered DASS subscale scores were entered at the first step, PAS and FNE scores at the second step, and the FNE x PAS interaction term at the third step.

3. Results 
Raw score means and standard deviations for FNE, PAS, DASS subscale, and PSQ subscale scores, as well as correlations among raw scores on all measures are presented in Table 2. To control for the effects of multiple comparisons, we set alpha at 0.005 . Regression results (see Table 3)

Results of the first regression analysis predicting PSQ Interpersonal Suspicion/Hostility score show that R differed significantly from zero at the first two steps, each of which significantly improved model fit, but not at step 3. Of the predictors in the full model at step 3, only DASS Stress subscale score, FNE score, and PAS score accounted independently for significant proportions of the variance. FNE score accounted independently for 11 percent of the variance, PAS score accounted independently for four percent.

The regression analysis predicting PSQ Negative Mood/Withdrawal score yielded similar omnibus results, with significantly improved model fit at each of the first two steps, but not at the third. For this dependent variable, however, only DASS Depression subscale score, FNE score, and PAS score accounted independently for significant variance proportions in the final model. PAS score and FNE score accounted independently for only modest proportions of the variance: two percent and four percent respectively.

When PSQ Anger/Impulsiveness score served as the dependent variable, R differed significantly from zero at all three steps and model fit was significantly improved at each of the first two steps, but not the third step. In the final model, DASS Stress subscale score and, unexpectedly, PAS score accounted for significant proportions of the variance, with PAS score independently contributing 5\%.

The regression analysis predicting PSQ Mistrust/Wariness score yielded significant results at the model level at all three steps, but model fit was only significantly improved at steps 
one and two. In the final model, significant independent contributions to the variance came only from the DASS Stress subscale score and FNE score, which each accounted for one percent.

PSQ Perceived Hardship/Resentment score served as the dependent variable in the final regression analysis; $\mathrm{R}$ differed significantly from zero and model fit was improved at each of the first two steps; R differed significantly from zero at the third step, but model fit was not significantly better than at the second step. In the final model, both DASS Stress and DASS Depression scores were significant independent predictors, along with both PAS score, which accounted independently for two percent of the variance, and FNE score, which accounted for an additional two percent.

\section{Discussion}

The present results provide partial support for our hypotheses. Consistent with predictions, both self-reported social anxiety and self-reported perceptual anomalies made significant independent contributions to scores on a multidimensional measure of paranoid ideation, even when current negative affect was covaried. Social anxiety, as measured by the self-report Fear of Negative Evaluation Scale, significantly predicted scores on PSQ factors labeled Interpersonal Suspiciousness/Hostility, Negative Affect/Withdrawal, Mistrust/Wariness, and Perceived Hardship/Resentment; experience of perceptual anomalies significantly predicted scores on all factors except Mistrust/Wariness. Contrary to our expectations, however, the interaction of social anxiety and perceptual aberration was not a significant predictor of any aspect of normal range paranoid thought.

Broadly, these findings suggest that social anxiety and the experience of perceptual anomalies both can play parts in the emergence of sub-clinical paranoid patterns of thinking. Our results do not, however, provide support for the idea that they operate in an interactive manner, 
such that the experience of perceptual anomalies, which are prone to negative, self-referential interpretation, increases risk of paranoid thought in socially anxious individuals, who are likely to make such interpretations. Instead, social anxiety and perceptually aberrant experiences appear to contribute distinctly and independently to different aspects of paranoid thinking.

Our data suggest that social anxiety is most strongly associated with scores on the Interpersonal Suspiciousness/Hostility factor of the PSQ, accounting independently for 11 percent of the variance. Items within this factor tap negative self-referential beliefs (e.g., that others are likely to watch, talk about, insult, or take advantage of you) that are consistent with common cognitions among the socially anxious; the strong association with fear of negative evaluation is thus not unexpected. FNE scores also, along with DASS Depression scores, accounted for significant portions of the variance in scores on the PSQ Negative Mood/Withdrawal factor. Thus, as we predicted, the Negative Mood/Withdrawal scale appears to tap global negative affect; although such negative affect may accompany perceptual anomalies, it is also likely to be common in their absence.

The associations between FNE scores and scores on the Perceived Hardship/Resentment factor, which reflects feelings of being put upon or mistreated by others or by fate, and the Mistrust/Wariness factor, are weaker (FNE accounts independently for only two percent of the variance in Perceived Hardship/Resentment scores and one percent of the variance in Mistrust/Wariness scores) and less readily explained. The tendency among socially anxious individuals to engage in self-critical rumination, especially after anxiety-provoking interactions (Clark \& Wells, 1995), might bias them to recall interpersonal experiences in a negative light, particularly when they look ahead to future interactions. They may thus selectively remember occasions when they felt taken advantage of and overlook occasions when events unfolded 
favorably for them. Alternatively or additionally, social anxiety-both fear of negative evaluation and proclivity toward social avoidance-may genuinely be associated with unfair treatment at the hands of others that legitimately evokes mistrust. Indeed, the experience of peer victimization has been linked to social anxiety in youths (Storch et al., 2003) and adults (Zapf et al., 2011).

Consistent with prior research that treated paranoia as a unitary construct (Freeman et al., 2008a), the experience of perceptual anomalies emerged as a modest but significant independent predictor of scores on all PSQ factors except the Mistrust/Wariness factor, suggesting that individuals who experience perceptual aberrations are at heightened risk for feeling not only suspicious and resentful of others, but also for feeling angry and generally distressed. Given the common co-occurrence of perceptual anomalies and paranoid patterns of thought in individuals with disorders on the schizophrenia spectrum and those with borderline personality characteristics (APA, 2000), these findings might be driven by a subgroup of participants with undiagnosed or subthreshold mental illness. Alternatively, recent research exploring the role of maladaptive metacognitive processes in mediating associations among perceptual anomalies, emotional distress and other negative outcomes may shed light on our findings. Brett and colleagues (2009) found evidence that interactions between maladaptive metacognitions and perceptual aberrations may yield clinically significant psychotic symptoms; such interactions could lead to paranoid thoughts and associated negative affect.

We included DASS subscales scores as covariates at the first steps in all models to provide a more stringent test of the specificity of any observed effects for our core variables of interest. Notably, broad dimensions of negative affect, as reflected by these scores, accounted independently for reasonable proportions of the variance in some PSQ subscale scores, 
particularly, and not surprisingly, the Negative Mood and Anger/Impulsiveness subscales. Our findings that FNE and PAS scores made significant independent contributions to several factor scores above and beyond those of the DASS predictors is thus striking.

Our central findings, in a sample of relatively high-functioning college students, are loosely consistent with Freeman and colleagues’s findings that the combination of anxiety and the experience of perceptual anomalies increases the risk that an individual will report feeling persecuted (Freeman et al., 2005a; Freeman et al., 2008a; Freeman et al., 2008b). However, the effects of anxiety and perceptual aberrations appear to be independent and additive, with each making distinct, if subtle, contributions to different aspects of paranoid thinking. Our findings are thus inconsistent with Freeman and colleagues’ proposal that increased risk of paranoid thought stems from a heightened tendency in individuals with elevated negative affect, at least anxiety about social situations, to interpret anomalous perceptual experiences as persecutory. It is possible that perceptual anomalies interact significantly with more broadly generalized negative affect; inclusion of a measure of typical, rather than current, experiences of negative emotion would have facilitated tests of this hypothesis and future studies would benefit from sampling a larger range of negative emotional experiences.

Our study is limited by several issues. First, we collected only self-report data. Ideally, we would have augmented our self-report instruments with other-report and/or observational measures to decrease the possibility that our findings reflect method-related factors rather than latent trait-related factors. Inclusion of such additional measures, as well as self-report infrequency measures, would also have provided more objective characterizations of our participants, whose self-reports may have been biased by the very characteristics that we asked them to describe in themselves or by reluctance to endorse experiences that we asked them to 
document (e.g., psychological treatment history). Collection of such data, however, would have been prohibitive in a sample of this size; in future research, we aim to replicate our findings in smaller groups on whom we collect both subjective and objective measures of both predictor and dependent variables.

A second primary limitation of our study is the use of a heavily female college-student sample, which decreases generalizability to the population at large. Results from our sample are also potentially limited in that, although we went to great lengths to ensure participant anonymity, wariness about repercussions of self-disclosure could have influenced responses in at least a subset of participants. Consistent with this concern, although most participants completed all measures fully, a sizable number $(n=15)$ of participants declined to complete the PAS; additional participants skipped items that tapped highly atypical behaviors or thoughts and were thus excluded from all analyses. The participants who skipped items or declined to complete the PAS did not differ according to demographic variables from those who completed all items; however, we may have failed to measure dimensions that set these two groups apart.

Despite these limitations, our results add to the extant literature by providing evidence in a large, racially diverse, non-clinical sample, that social anxiety and perceptual aberrations make significant contributions to the prediction of normal-range paranoid ideation, even when general negative affect is covaried. They appear, however, to operate in an additive, rather than an interactive manner, contrary to our predictions. In addition to the future directions we have suggested above, we encourage research aimed at examining whether anxiety more generally, as well as other types of negative affect such as depression, might interact with perceptual anomalies to engender paranoid responses. To this end, creative studies such as Freeman et al.'s 
(2008) experiment in which the authors assessed state paranoia and social anxiety after participants experienced a neutral virtual reality environment are likely to be fruitful. 


\section{References}

American Psychiatric Association APA, 2000. Diagnostic and Statistical Manual of Mental Disorders, Fourth Edition, Text Revision (DSM-IV-TR), Author, Washington, D.C.

Beard, C., Amir, N., 2009. Interpretation in Social Anxiety: When Meaning Precedes Ambiguity. Cognitive Therapy and Research 33, 406-415.

Beck A.T., Steer R.A., Brown G.K., 1996. Beck Depression Inventory Manual, 2nd ed. Psychological Corporation, San Antonio TX.

Brett, C.M.C., Johns, L.C., Peters, E.P., McGuire, P.K., 2009. The role of metacognitive beliefs in determining the impact of anomalous experiences: a comparison of helpseeking and non-help-seeking groups of people experiencing psychotic-like anomalies. Psychological Medicine 39, 939-950.

Chadwick, P.D.J., Trower, P., 1997. To defend or not to defend: A comparison of paranoia and depression. Journal of Cognitive Psychotherapy 11, 63-71.

Chapman, J.P., Chapman, L.J., Kwapil, T.R., 1995. Scales for the measurement of schizotypy. In Raine, A., Lencz, T., Mednick, S.A. (Eds.), Schizotypal Personality. Cambridge University Press, New York, pp. 79-106.

Chapman, L.J., Chapman, J.P., Raulin, M.L., 1978. Body-image aberration in schizophrenia. Journal of Abnormal Psychology 87, 399-407.

Clark, D.M., Wells, A., 1995. A cognitive model of social phobia. In Heimberg, R.G., Liebowitz, M.R., Hope, D.A., Schneier, F.R. (Eds.), Social Phobia: Diagnosis, Assessment, and Treatment. Guilford Press, New York, pp. 69-93.

Combs, D.R., Penn, D.L., Chadwick, P., Trower, P., Michael, C.O., Basso, M.R., 2007. Subtypes of paranoia in a nonclinical sample. Cognitive Neuropsychiatry 12, 537-553.

Crawford, J.R., Henry, J.A., 2003. The Depression Anxiety Stress Scales (DASS): Normative data and latent structure in a large non-clinical sample. British Journal of Clinical Psychology 42, 111-113.

Ellett, L., Lopes, B., Chadwick, P., 2003. Paranoia in a Nonclinical Population of College Students. Journal of Nervous and Mental Disease 191, 425-430.

Fenigstein, A., Vanable, P.A., 1992. Paranoia and self-consciousness. Journal of Personality and Social Psychology 62, 129-138.

Fornells-Ambrojo, M., Garety, P.A., 2009. Understanding attributional biases, emotions and self-esteem in "poor me" paranoia: Findings from an early psychosis sample. British Journal of Clinical Psychology 48, 141-162. 
Freeman, D., 2007. Suspicious minds: The psychology of persecutory delusions. Clinical Psychology Review 27, 425-457.

Freeman, D., Garety, P.A., Bebbington, P., Slater, M., Kuipers, E., Fowler, D., Green, C., Jordan, J., Ray, K., Dunn, G., 2005a. The Psychology of Persecutory Ideation II: A Virtual Reality Experimental Study. Journal of Nervous and Mental Disease 193, 309315.

Freeman, D., Garety, P.A., Bebbington, P.E., Smith, B., Rollinson, R., Fowler, D., Kuipers, E., Ray, K., Dunn, G., 2005b. Psychological investigation of the structure of paranoia in a non-clinical population. British Journal of Psychiatry 186, 427-435.

Freeman, D., Gittins, M., Pugh, K., Antley, A., Slater, M., Dunn, G., 2008a. What makes one person paranoid and another person anxious? The differential prediction of social anxiety and persecutory ideation in an experimental situation. Psychological Medicine 38, 1121-1132.

Freeman, D., Pugh, K., Antley, A., Slater, M., Bebbington, P., Gittins, M., Dunn, G., Kuipers, E., Fowler, D., Garety, P., 2008b. Virtual reality study of paranoid thinking in the general population. British Journal of Psychiatry 192, 258-263.

Garety, P.A., Freeman, D., 1999. Cognitive approaches to delusions: A critical review of theories and evidence. British Journal of Clinical Psychology 38, 113-154.

Green, C.E.L., Freeman, D., Kuipers, E., Bebbington, P., Fowler, D., Dunn, G., Garety, P.A., 2008. Measuring ideas of persecution and social reference: The Green et al. Paranoid Thought Scales (GPTS). Psychological Medicine 38, 101-111.

Gudjonsson, G.H., Sigurdsson, J.F., Brynjólfsdóttir, B., Hreinsdóttir, H., 2002. The relationship of compliance with anxiety, self-esteem, paranoid thinking and anger. Psychology, Crime \& Law 8, 145-153.

Hofmann, S.G., Heinrichs, N., Moscovitch, D.A., 2004. The nature and expression of social phobia: Toward a new classification. Clinical Psychology Review 24, 769-797.

Kwapil, T.R., Barrantes-Vidal, N., Silvia, P.J., 2008. The dimensional structure of the Wisconsin Schizotypy Scales: Factor identification and construct validity. Schizophrenia Bulletin 34, 444-457.

Langdon, R., Coltheart, M., 2000. The cognitive neuropsychology of delusions. Mind and Language 15, 184-218.

Lenzenweger, M.F., 1994. Psychometric high-risk paradigm, perceptual aberrations, and schizotypy: An update. Schizophrenia Bulletin 20, 121-135.

Lincoln, T.M., Peter, N., Schafer, M., Moritz, S., 2009. Impact of stress on paranoia: An experimental investigation of moderators and mediators. Psychological Medicine 39, 1129-1139. 
Lovibond P.F., Lovibond S.H., 1995. The structure of negative emotional states: comparison of the depression anxiety stress scales (DASS) with the Beck depression and anxiety inventories. Behaviour Research and Therapy 33, 335-343.

Maher, B.A., 1974. Delusional thinking and perceptual disorder. Journal of Individual Psychology 30, 98-113.

Martin, J.A., Penn, D.L., 2001. Social cognition and subclinical paranoid ideation. British Journal of Clinical Psychology 40, 261-265.

Meyer, E.C., Lenzenweger, M.F., 2009. The specificity of referential thinking: A comparison of schizotypy and social anxiety. Psychiatry Research 165, 78-87.

Niewenhujisen K., de Boer A.G., Verbeek J.H., Blonk R.W., van Dijk F.J., 2003. The Depression Anxiety Stress Scales (DASS): detecting anxiety disorder and depression in employees absent from work because of mental health problems. Occupational and Environmental Medicine 60, 77-82.

Orsillo, S.M., 2001. Measures for social phobia. In M. M. Antony, S. M. Orsillo, \& E. Roemer (Eds.), Practitioner's guide to empirically based measures of anxiety. Kluwer, New York, pp. 165-187.

Pollard, C.A., Henderson, J.G., 1988. Four types of social phobia in a community sample. Journal of Nervous and Mental Disease 176, 440-445.

Rawlings, D., Freeman, J.L., 1996. A questionnaire for the measurement of paranoia/suspiciousness. British Journal of Clinical Psychology 35, 451-561.

Rodebaugh, T.L., Woods, C.M., Thissen, D.M., Heimberg, R.G., Chambless, D.L., Rapee, R.M., 2004. More information from fewer questions: The factor structure and item properties of the original and brief Fear of Negative Evaluation Scale. Psychological Assessment 16, 169-181.

Storch, E.A., Brassard, M.R., Masia-Warner, C.L., 2004. The relationship of peer victimization to social anxiety and loneliness in adolescence. Journal of Adolescence 27, 351-362.

Sukantarat, K.T., Williamson, R.C., Brett, S.J., 2007. Psychological assessment of ICU survivors: a comparison between the Hospital Anxiety and Depression scale and the Depression, Anxiety and Stress scale. Anaesthesia 62, 239-243.

Thewissen, V., Myin-Germeys, I., Bentall, R., de Graaf, R., Vollebergh, W., van Os, J., 2007. Instability in self-esteem and paranoia in a general population sample. Social Psychiatry and Psychiatric Epidemiology 42, 1-5.

Watson, D., Friend, R., 1969. Measurement of social-evaluative anxiety. Journal of Consulting \& Clinical Psychology 33, 448-457. 
Zapf, D., Einarson, S., 2011. Individual antecedents of bullying: Victims and perpetrators. In S. Einarson, H. Hoel, D. Zapf, C.L. Cooper (Eds.), Bullying and Harassment in the Workplace: Developments in Theory, Research and Practice, Second Edition. CRC Press, Boca Raton, Florida, pp. 177-200. 